\title{
Breaking through the surface: more to learn about lipids and cardiovascular disease
}

\author{
Justin B. Echouffo-Tcheugui, ${ }^{1}$ Mohit Jain, ${ }^{2,3}$ and Susan Cheng ${ }^{4}$ \\ 'Division of Endocrinology, Diabetes and Metabolism, Department of Medicine, Johns Hopkins University School of Medicine, Baltimore, Maryland, USA. ${ }^{2}$ Department of Medicine and ${ }^{3}$ Department of \\ Pharmacology, School of Medicine, UCSD, La Jolla, California, USA. "Smidt Heart Institute, Cedars-Sinai Medical Center, Los Angeles, California, USA.
}

\begin{abstract}
The human lipidome comprises over tens of thousands of distinct lipid species in addition to total cholesterol and the other conventional lipid traits that are routinely measurable in the peripheral circulation. Of the lipid species considered to exhibit bioactive functions, sphingolipids are a class of molecules that have shown relevance to human disease risk and cardiovascular outcomes in particular. In this issue of the JCI, Poss et al. conducted targeted lipidomics in a case-control study involving over 600 individuals and found a sphingolipid profile that predicted coronary artery disease status. In the context of emerging evidence linking sphingolipid biology with cardiovascular pathophysiology, these results suggest the potential utility of serum sphingolipids as cholesterol-independent markers of risk and even future targets for optimizing cardiovascular health.
\end{abstract}

\section{Bioactive lipids}

The human lipidome comprises over tens of thousands of distinct lipid species, many of which have primarily structural or energetic functions and others of which play a variety of roles in cellular regulatory pathways (1). The latter lipids, which regulate responses to a diversity of specific stimuli, are termed bioactive lipids. Sphingolipids are a family of bioactive lipids that have emerged as molecules of special interest (Figure 1). Distinguished biochemically by the presence of a sphingoid base, sphingolipids were originally named in the early 20th century after the mythical Sphinx creature, given their enigmatic nature (1). Now, sphingolipids are recognized to be involved in a wide range of functions including, but not limited to, the regulation of cell-cell interactions, cellular growth, migration, apoptosis, senescence, and inflammatory responses. In turn, as key participants of all major cell signaling pathways, sphingolipids and their metabolism have also been implicated in the risk for developing a variety of human diseases including neurodegenerative, metabolic, oncologic, immunologic, and cardiovascular disease conditions.

The links between sphingolipids, including the ceramide family of lipids, and cardiovascular disease traits have garnered particular attention. Prior human studies have found higher plasma ceramide levels and lower sphingosine-1phosphate levels in patients with unstable angina or myocardial infarction, as well as in the setting of hypertension and type 2 diabetes (2). Although the biology underlying these observations remains unclear, mechanistic studies have implicated ceramides in atherosclerotic processes (3), such as lipoprotein aggregation, uptake of lipoproteins and accumulation of cholesterol within macrophages, regulation of nitric oxide synthesis, production of super-

Related Article: p. 1363

Conflict of interest: SC reports receiving consulting fees from Zogenix.

Copyright: @ 2020, American Society for Clinical Investigation.

Reference information: J Clin Invest. 2020;130(3):1084-1086. https://doi.org/10.1172/JCI134696.

oxide anions, and expression of various cytokines (4). In addition, sphingosine1-phosphate has been shown to regulate vasoconstriction and vasodilation, sodium excretion in the renal medulla, and pancreatic $\beta$ cell production of insulin $(2,5)$. In this context, a number of clinical studies have explored the extent to which sphingolipids might account for the residual risk not explained by conventional lipid traits and subfractions (i.e., total cholesterol, LDL cholesterol, and HDL cholesterol) in models predicting outcomes such as coronary artery disease (CAD) (6). Certain ceramides, in particular, have been shown to offer incremental predictive value over the routinely used lipid markers and other conventional risk markers for CAD (7-9).

\section{Sphingolipids in relation to CAD}

Extending their research from prior work, Poss et al. (10) report in this issue of the JCI intriguing results from a case-control study of sphingolipids and CAD. The investigators used a targeted liquid chromatography mass spectrometry method to examine a panel of 32 sphingolipids in relation to CAD in a single-center study of 462 patients and 212 population-based control subjects. Of the sphingolipids measured, 30 were significantly elevated in patients with CAD compared with controls. Sphingosine, dihydro-cer(d18:0/16:0), dihydrocer(d18:0/18:0), and cer(d18:1/24:1) were most strongly associated with CAD in adjusted models, with ORs for CAD at least twice those for LDL cholesterol (11). The investigators then generated a sphingolipidinclusive CAD (SIC) risk score, which included all the sphingolipids observed in relation to CAD and appeared to estimate CAD status independently and more effectively than conventional risk factors (including total cholesterol, LDL cholesterol, and HDL cholesterol, as well as serum triglycerides). The 
A Individual biochemical diversity

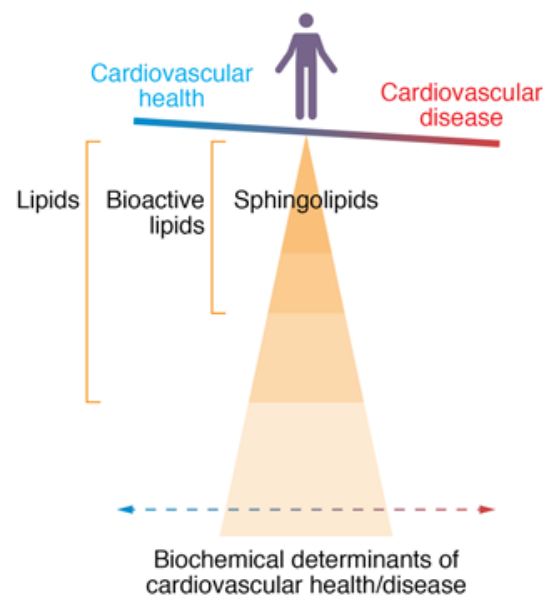

B Population biochemical diversity

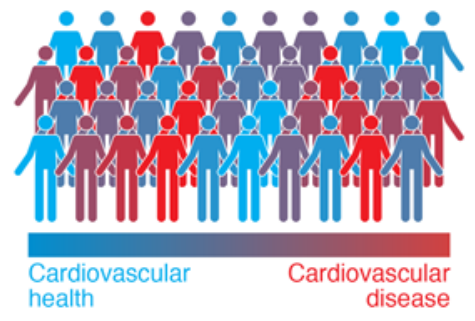

Figure 1. Bioactive lipids and cardiovascular

risk. The human lipidome includes thousands of bioactive lipids, of which sphingolipids represent a class with particular relevance to the balance between cardiovascular health and disease.
SIC risk score also performed better at predicting CAD than did a previously defined and exclusively ceramide-based risk score, the cardiac event risk test 1 (CERT1). In these analyses, the incremental predictive value of the SIC score was strong and significant according to the C-statistic, net reclassification index, and integrated discrimination improvement metrics.

The finding that certain low-abundance, rather than other high-abundance, ceramides appeared important for determining CAD risk is a key observation. As the authors point out, some of the more abundant but less predictive ceramides are known to drive tissue and metabolic function. Similar to the phenomenon of housekeeping genes, more-abundant bioactive lipids may be required for maintaining basic cellular processes; thus, although potentially essential for function, circulating levels of these more-abundant lipids are less likely to inform or distinguish disease states. By contrast, certain less-abundant lipid species may represent more dynamic processes that vary between individuals in ways that ultimately drive clinically relevant pathophysiology. Poss et al. (10) surmise that such processes could involve relative metabolic flux in the levels of precursor and product species, even though ratiometric findings failed to emerge from their analyses. It is possible that potentially important ratiometric patterns are not discernible from peripherally circulating, less tissue-specific measures of sphingolipids - or that more sensitive methods are needed to detect a wider range of less-abundant species to provide more granular information.
Another noteworthy aspect of the report by Poss et al. (10) is the study design. Analyses were limited to a singlecenter, case-control analysis performed on a fairly homogenous cohort of adults living among the predominantly Mormon community of Utah, without any external validation. Nonetheless, the relatively younger age of CAD onset among the patients provides a potentially greater specificity of results, and the total number of cases with 2:1 matching of controls offered adequate power to detect associations. Moreover, the authors elected to apply two complementary and advanced statistical methods: least absolute shrinkage and selection operator (LASSO) regression and random forest analysis (RFA). This approach to data analyses allowed both a sparse method for analyzing multiple potential predictor sphingolipids at once and a method for not assuming linearity of associations between the predictors and the outcome. In effect, although LASSO models may not perform well for multiple putative predictor molecules that could be highly intercorrelated, RFA models are not as susceptible to this issue; in turn, although RFA models are less efficient if associations are truly linear, LASSO models will optimally accommodate for such linearity (12). It should be noted, however, that these two methods are not the only nontraditional or machine-learning methods that can be applied to these types of high-dimensional data, for which ensemble approaches could be considered in the future.

\section{Clinical implications}

With regard to the prospect of translating discovery in bioactive lipids to an application for clinical practice, this report by Poss et al. offers a step forward (10). Compared with the CERT1 ceramide plasma test, commercially released in 2016 by the Mayo Medical Laboratories for evaluation of cardiac risk (13), the broader SIC risk score captures a wider scope of potentially variable sphingolipid biology $(7-9,13)$ and performs better in the index cohort described in the study by Poss et al. However, further work is needed to validate the ability of the same or a similar set of biomarkers to perform in prospective analyses and in populations more diverse, across a number of characteristics including age, sex, race/ethnicity, and comorbid status (14). Future investigations could also consider expanding the reach of profiling methods to detect a more complete range of low-abundance sphingolipids including more species potentially associated with protection rather than harm, especially if we believe that biological flux in sphingolipid metabolism is relevant. Most important, from a clinical perspective, the efficacy and cost-effectiveness of directing interventions on the basis of any type of sphingolipid risk score remain unknown. Nonetheless, although there is still much to learn about sphingolipid biology and its role in modulating cardiovascular risk, the study by Poss et al. (10) augments an expanding body of evidence pointing to the sphingolipids as bioactive lipid molecules of relevance to the balance of human health and disease. 


\section{Acknowledgments}

This work was supported in part by grants from the NIH (R01-HL134168 and R01HL143227, to SC and MJ); the American Heart Association (CVGPS Pathway Award, to SC and MJ); and the Doris Duke Charitable Foundation (to MJ and SC).

Address correspondence to: Justin B. Echouffo-Tcheugui, Division of Endocrinology, Diabetes and Metabolism, Department of Medicine, Johns Hopkins School of Medicine, 5501 Hopkins Bayview Circle, Baltimore, Maryland 21204, USA. Phone: 410.550.3054; Email: jechouf1@jhmi.edu.

1. Hannun YA, Obeid LM. Sphingolipids and their metabolism in physiology and disease. Nat Rev Mol Cell Biol. 2018;19(3):175-191.

2. Borodzicz S, Czarzasta K, Kuch M, CudnochJedrzejewska A. Sphingolipids in cardiovascular diseases and metabolic disorders. Lipids Health Dis. 2015;14:55
3. Schissel SL, Tweedie-Hardman J, Rapp JH, Graham G, Williams KJ, Tabas I. Rabbit aorta and human atherosclerotic lesions hydrolyze the sphingomyelin of retained low-density lipoprotein. Proposed role for arterial-wall sphingomyelinase in subendothelial retention and aggregation of atherogenic lipoproteins. J Clin Invest. 1996;98(6):1455-1464.

4. Bismuth J, Lin P, Yao Q, Chen C. Ceramide: a common pathway for atherosclerosis? Atherosclerosis. 2008;196(2):497-504.

5. Shahin MH, et al. Sphingolipid metabolic pathway impacts thiazide diuretics blood pressure response: insights from genomics, metabolomics, and lipidomics. J Am Heart Assoc. 2017;7(1):e006656.

6. Matsuura Y, Kanter JE, Bornfeldt KE. Highlighting residual atherosclerotic cardiovascular disease risk. Arterioscler Thromb Vasc Biol. 2019;39(1):e1-e9.

7. Laaksonen $\mathrm{R}$, et al. Plasma ceramides predict cardiovascular death in patients with stable coronary artery disease and acute coronary syndromes beyond LDL-cholesterol. Eur Heart J. 2016;37(25):1967-1976.

8. Hilvo M, et al. Development and validation of a ceramide- and phospholipid-based cardiovascular risk estimation score for coronary artery disease patients [published online ahead of print June 18, 2019]. Eur Heart J. https://doi. org/10.1093/eurheartj/ehz387.

9. Peterson LR, et al. Ceramide remodeling and risk of cardiovascular events and mortality. JAm Heart Assoc. 2018;7(10):e007931.

10. Poss AM, et al. Machine learning reveals serum sphingolipids as cholesterol-independent biomarkers of coronary artery disease. J Clin Invest. 2020;130(3):1363-1376.

11. Sniderman $\mathrm{AD}$, et al. A meta-analysis of low-density lipoprotein cholesterol, non-high-density lipoprotein cholesterol, and apolipoprotein B as markers of cardiovascular risk. Circ Cardiovasc Qual Outcomes. 2011;4(3):337-345.

12. Antonelli J, et al. Statistical workflow for feature selection in human metabolomics data. Metabo lites. 2019;9(7):E143.

13. Nicholls M. Plasma ceramides and cardiac risk. Eur Heart J. 2017;38(18):1359-1360.

14. Moons KG, et al. Risk prediction models: II. External validation, model updating, and impact assessment. Heart. 2012;98(9):691-698. 